\title{
Anomalous Spot Abundances in the CMB
}

\author{
Maik Weber*, Youness Ayaita \\ Institut für Theoretische Physik, Universität Heidelberg, Philosophenweg 16, Heidelberg, \\ D-69120, Germany \\ E-mail: m.weber@thphys . uni-heidelberg.de
}

In this talk, we present the main results of an analysis of large-angular hot and cold spot abundances in the five-year temperature anisotropy maps of WMAP. In comparison with Gaussian simulations based on the $\Lambda \mathrm{CDM}$ best-fit model, too few spots are detected in the cut-sky maps (outside the KQ75 mask). We find that this is linked to an anomalously low value of the observed mean temperature fluctuation on the considered scales. Further, a study of the ILC full-sky map reveals an intriguing overdensity of spots inside the usually masked region. We discuss the relation between our results and the lack of large-scale power as inferred from the low- $\ell$ multipoles or the correlation function at large angles. The talk is entirely based on [1], where a thorough presentation of our algorithm and many more details can be found.

International Workshop on Cosmic Structure and Evolution - Cosmology2009,

September 23-25, 2009

Bielefeld, Germany

${ }^{*}$ Speaker. 


\section{Introduction}

Observations of the Cosmic Microwave Background (CMB) probe cosmology over a large range of scales. As a snapshot of the Universe at the age of decoupling, it provides us with a wealth of information about the physics of the early Universe, the stochastic properties and the spectrum of the primordial fluctuations. Furthermore, the late-time formation of large-scale structure leaves insightful imprints in the CMB anisotropy pattern via the integrated Sachs-Wolfe (ISW) effect and higher-order contributions [2]. After five years of observation, the Wilkinson Microwave Anisotropy Probe (WMAP) provides the best CMB data sets available today. It has helped to establish the simplest six-parameter $\Lambda \mathrm{CDM}$ model as the concordance model of modern cosmology. Assuming Gaussian fluctuations, the statistical information is encoded in the angular power-spectrum, which is in good agreement with $\Lambda \mathrm{CDM}$ predictions apart from the well-known quadrupole anomaly $[3,4]$. Though, several authors have found intriguing anomalies on largeangular scales when going beyond harmonic representation, e.g. the planarity and alignment of low- $\ell$ multipoles [5, 6], the vanishing of the correlation function above $\sim 60^{\circ}$ [7] or the famous Cold Spot in the southern hemisphere [8]. Although cosmic variance strictly limits the significance of any of these findings, the accumulation of many such large-scale peculiarities may indicate deviations from the simple concordance model, either concerning the primordial fluctuations or late-time processes that may be related to the dynamics of dark energy.

We have investigated the abundances of large-angular hot and cold spots in the WMAP-5 temperature anisotropy maps and developed an intuitive algorithm for this task. It smoothly scans through the sky maps and determines the mean temperature contrast in extended regions of a selected scale. A variable temperature threshold defines the regions which are counted as hot and cold spots in a map. The numbers can be directly compared to Gaussian simulations. Our results reveal striking discrepancies, which we will briefly summarize in this talk. For more details on the implementation of the algorithm and a detailed discussion of the results, we refer the reader to [1]. Other authors, who have investigated local extrema in the CMB temperature anisotropy field, also find deviations from the concordance model $[11,12,13]$, which may be related to our findings.

\section{Method}

Sky maps of the CMB assign a temperature anisotropy $\Delta T(\theta, \varphi)$ to each direction $\mathbf{n}=(\theta, \varphi)$. When searching for spots in these maps, we define their shape via a window function $W(\theta, \varphi)$, which essentially allows to average the temperature anisotropies over the region of consideration,

$$
\Delta T_{W}=\int d \Omega \Delta T(\theta, \varphi) W(\theta, \varphi) .
$$

The mean temperature contrast $\Delta T_{W}$ of a region is then compared to a threshold $\Delta \mathfrak{T} \geq 0$. We call the region a hot spot, if $\Delta T_{W} \geq \Delta \mathfrak{T}$ and a cold spot, if $\Delta T_{W} \leq-\Delta \mathfrak{T}$. Typically, $\Delta \mathfrak{T}$ is varied over the whole range of interest. The number of detected spots usually decreases with increasing thresholds until it eventually falls to zero, when the threshold exceeds the maximum temperature contrast of spots in a map. A characteristic scale for the threshold can be obtained by taking the average of the mean temperature contrasts in regions of the chosen shape,

$$
\Delta T_{\mathrm{rms}}=\sqrt{\left\langle\Delta T_{W}^{2}\right\rangle} .
$$


By virtue of the ergodic theorem, and as long as the investigated scales are not too large, the ensemble average can be replaced by the average over distinct regions on the sphere. This allows us to measure $\Delta T_{\text {rms }}$ in a map.

The WMAP team provides foreground-reduced sky maps for three different frequency bands $(\mathrm{Q}, \mathrm{V}, \mathrm{W})$ and corresponding differencing assemblies ${ }^{1}$. Since the V-band map is less foreground contaminated than the Q-band map and exhibits a better signal-to-noise ratio than the W-band map [3], we obtain our main results from this map (the others have been checked for consistency). As is common practice for temperature analyses, we apply the conservative KQ75 mask to exclude highly contaminated and thus unreliable regions of the map from our investigation. The residual monopole and dipole are removed afterwards. For this task and many others, we employ the comprehensive HEALPix facilities [14].

Our algorithm primarily searches for spots in the observed cut-sky maps. Robust theory predictions can be obtained from a large number of Gaussian simulations with WMAP beam and noise properties. The theory input is then solely given by the angular power spectrum $C_{\ell}$, defined via the two-point correlators of the coefficients $a_{\ell m}$ of the spherical harmonics decomposition of the temperature anisotropy field,

$$
\left\langle a_{\ell m} a_{\ell^{\prime} m^{\prime}}^{*}\right\rangle=\delta_{\ell \ell^{\prime}} \delta_{m m^{\prime}} C_{\ell}
$$

As common, we have assumed statistical isotropy above. We aim at a comparison with the concordance model, the six parameter $\Lambda \mathrm{CDM}$ model. The current best-fit $C_{\ell}$ are obtained from the legacy archive, together with the noise and beam properties of WMAP.

A basic requirement of the algorithm consists in dividing the sphere into sectors that allow for a smooth scanning through the map. These sectors are demanded to have equal surface area $A$ (we use the parameter $a=\sqrt{A}$ to specify the scale of investigation). The actual shape of the sectors turns out to be marginal (at least as long as we take care for regular shapes to keep small-scale influences small), hence we do not impose exactly the same shapes all over the sphere. A convenient choice are intersections of longitude and latitude rings, since they easily allow for a smooth scanning through the maps by slightly changing their boundary angles. Further, these intersections provide clear boundaries, which makes it easy to avoid multiple counting of spots (the algorithm ignores spots overlapping with previously found ones).

For a given threshold $\Delta \mathfrak{T}$, the algorithm scans the observed cut-sky map and the Gaussian simulations by smoothly varying the boundary angles of the sectors over their whole ranges. A slight adaption also allows to measure $\Delta T_{\text {rms. }}$. For this task, the algorithm jumps from one sector to another rather than smoothly scanning through all possible ones. The average temperature contrast in the sectors gives $\Delta T_{\text {rms }}$ of the scale $a$ in the map. For all these applications, we allow for a slight overlap with the mask (maximally $5 \%$ of masked pixels inside a sector) to achieve good statistics. The masked pixels are assigned zero temperature contrast and thus cannot lead to misinterpreted spots. The comparison between observation and theory is not significantly affected, since both, observed and simulated maps, are treated in exactly the same way.

\footnotetext{
${ }_{1}$ available on the legacy archive http://cmbdata.gsfc.nasa.gov
} 


\section{Results}

\subsection{Observation and $\Lambda$ CDM Expectations}

We compare the observed spot abundances and the $\Lambda \mathrm{CDM}$ expectations on a representative angular scale of $a=6^{\circ}$ in Fig. 1. At low thresholds $\Delta \mathfrak{T}$, the numbers of spots in observed and
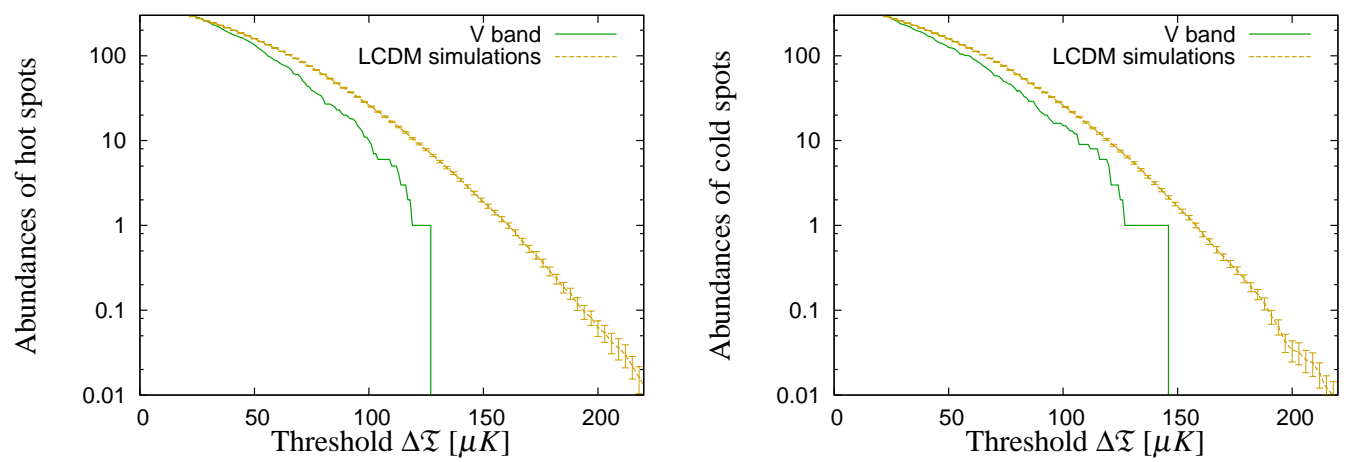

Figure 1: Observed and expected abundances of hot (on the left-hand side) and cold (on the right-hand side) spots in the CMB sky on an angular scale of $a=6^{\circ}$. The green curves show the spot abundances in the WMAP-5 V-band map, the orange ones show the mean spot abundances in 500 Gaussian $\Lambda$ CDM simulations with statistical errors.

simulated maps agree well, which has no physical meaning (almost any sector will exceed the close-to-zero threshold). Going to higher thresholds, where only significantly cold or hot regions are counted, the numbers of observed spots lies below the theoretical curves. The deviation is apparently less drastic for cold spots, where a single spot reaches a threshold close to $\sim 150 \mu \mathrm{K}$ (it is indeed located in the region of the Vielva Cold Spot, but still not exceeding the $\Lambda$ CDM expectation in our analysis).

We can quantify the lack of spots by choosing a simple estimator. The integrated spot abundance $s$ is defined as the sum of counted spots $n_{i}$ at the thresholds $i$ above the characteristic scale $\Delta \bar{T}_{\text {rms }}$ (obtained from the simulations),

$$
s \equiv \sum_{\Delta \mathfrak{T} \geq \Delta \bar{T}_{\mathrm{rms}}} n_{i}
$$

We count the number of Gaussian simulations with a value of $s$ that is smaller than the one belonging to the observed map and find strikingly low values. Only $p_{s}^{\text {hot }}=0.2 \%$ of Gaussian simulations have less hot spots than observed. The corresponding value for cold spots is somewhat larger, $p_{s}^{\text {cold }}=1.8 \%$. By comparing the observed map with each single simulation, we avoid any complications due to cosmic variance or correlations between different thresholds.

The lack of spots is reflected in an anomalously low value of the observed $\Delta T_{\text {rms. }}$. Only $p=$ $0.6 \%$ of simulated $\Lambda \mathrm{CDM}$ skies show a smaller value than the one found in the V-band map on the scale $a=6^{\circ}$. This does not improve when looking at other (large) scales as shown in Table 1. 
Table 1: $p$ gives the fraction of Gaussian $\Lambda \mathrm{CDM}$ simulations with a $\Delta T_{r m s}$ smaller than found in the V-band map for varying angular scales $a$.

\begin{tabular}{c|ccccc} 
Scale $a$ & $4^{\circ}$ & $5^{\circ}$ & $6^{\circ}$ & $7^{\circ}$ & $8^{\circ}$ \\
\hline Fraction $p$ & $0.50 \%$ & $0.62 \%$ & $0.60 \%$ & $0.16 \%$ & $0.36 \%$
\end{tabular}

\subsection{Spots in the ILC full-sky map}

The Gaussian simulations fulfill statistical isotropy by construction, but the spots in the single CMB sky we are able to observe could be distributed unevenly. It would be very insightful to have a look behind the mask and determine the total number of spots present in the whole sky, which is a highly non-trivial task. The five-year ILC (Internal Linear Combination) map is currently the best estimate for the full-sky CMB signal [15], although the WMAP team warns against its use on scales below $10^{\circ}$. This uncertainty prevents us from any precision analysis of the full-sky CMB sky, and the results must be handled with care. In this section, we are mainly interested in the distribution of spots rather than their total number, that's why we construct Gaussian simulations from the modelindependent $C_{\ell}$ as actually observed by WMAP [4] rather than the fitted ones. After applying our spot searching algorithm to the ILC map and the simulations, we separately collect those spots that have at most slight overlap with the usually masked region and the complementary ones, which would usually be hidden by the mask (we loosely refer to these two groups as spots inside and outside the mask, respectively). The comparison of observed and expected spot abundances in both groups are shown in Fig. 2. Interestingly, we find $p_{s}^{\text {outside }}=7 \%$ for the spots outside the mask, but $p_{s}^{\text {inside }}=96 \%$ for the spots inside the mask. The result is interpreted as follows. Outside the mask, we find still too few spots in the observed map, although we have replaced the theory $C_{\ell}$ by the WMAP-5 extracted $C_{\ell}$. The number is not very significant, but could in principle be a sign of non-Gaussianity (in this case, the $C_{\ell}$ spectrum would not determine the expected spot abundances
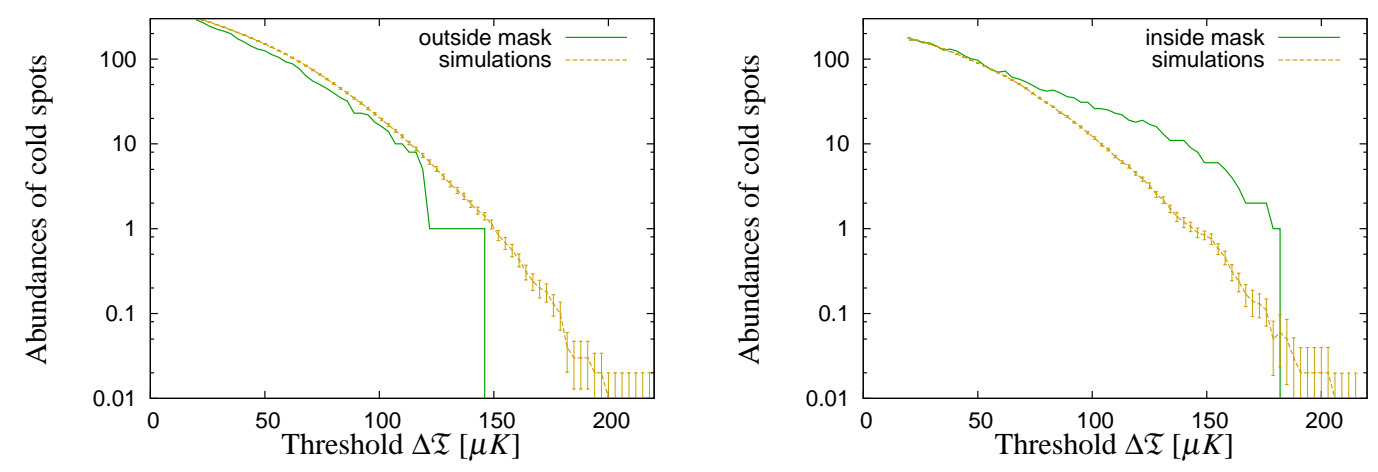

Figure 2: Spot abundances in the ILC map compared to simulations based on the measured WMAP-5 $C_{\ell}$ on an angular scale of $a=6^{\circ}$. The green plot on the left-hand side shows the number of spots that are detected in the region outside the KQ75 mask. On the right-hand side, the spots distributed in the complementary region are plotted. The orange curves show the mean spot abundances of Gaussian simulations in the corresponding regions. 
on their own). The second observation is even more striking: Inside the mask, the ILC map contains many more spots than expected. If confirmed by future CMB data, a highly anisotropic feature of the CMB sky could be a hint for statistical anisotropy in our Universe. Other authors also find evidence for anisotropy in the ILC map [7, 16, 17].

\subsection{Suppressing low- $\ell$ multipoles}

Lastly, we point out that our analysis does not suggest a violation of Gaussianity or statistical isotropy on its own. A suppression of power on large scales (small $\ell$ ) suffices in reconciling expectation and observation. This should not be seen as a re-estimation of the $C_{\ell}$, but it is insightful to see to which degree large-angular spot abundances and low- $\ell$ multipoles are related to each other. Motivated by the vanishing of the correlation function at degrees above $\sim 60^{\circ}$, we have modified the WMAP-5 extracted $C_{\ell}$ by setting $C_{\ell} \equiv 0$ for $\ell \leq 3$ (which is roughly linked to scales above $\left.60^{\circ}\right)$. Figure 3 shows that the values of $\Delta T_{\text {rms }}$ on large scales now agree well with observation. The same is true for the spot abundances, where we find $p_{s}^{\text {hot }}=30 \%$ and $p_{s}^{\text {cold }}=55 \%$ for Gaussian

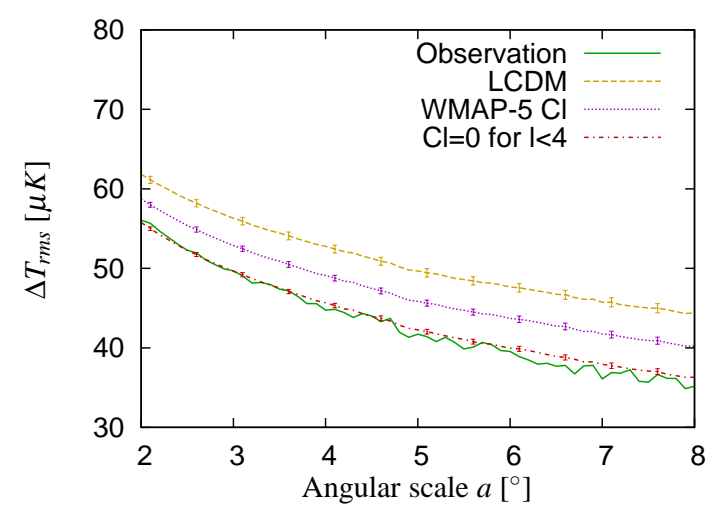

Figure 3: The mean temperature fluctuation for large angular scales $a$. We compare the observed values (obtained from the WMAP-5 V1 map) with theoretical expectations based on $\Lambda$ CDM simulations (top curve), simulations based on the WMAP-5 (a bit lower) and the modified spectrum (very close to the observation).

simulations based on the modified spectrum. Furthermore, we have checked that suppressing the low- $\ell$ multipoles by a substantial amount requires no fine-tuning in order to reconcile the data with expectation.

\section{Conclusions}

We have detected a lack of large-angular hot and cold spots in the WMAP-5 temperature anisotropy maps, that challenges the $\Lambda \mathrm{CDM}$ concordance model with Gaussian fluctuations at the $2 \sigma$ level. The discrepancies are intimately connected with an anomalously low mean temperature contrast on the scales of investigation. We have investigated the five-year ILC full-sky map to gain insights about the possible distribution of spots inside the usually masked region and found an intriguing overdensity of spots in this area. 
If not due to residual foregrounds or accepted as mere statistical coincidence, our results support a severe lack of power on large scales as already suggested by other works (e.g. [7]). A suppression of low- $\ell$ multipoles has considerably improved agreement with the current sky maps. Possible explanations for the anomalous spot abundances also include non-Gaussian signals and statistical anisotropy. These could encode important information on the physics of the early universe or on the late-time dynamics of the Universe. For instance, the CMB sky on large-angular scales could be dominated by very large structures in the local universe that might in principle resolve some of the well-known CMB anomalies $[9,10]$. Future CMB missions will probably shed some new light on the issues raised in this work.

\section{References}

[1] Y. Ayaita, M. Weber and C. Wetterich, "Too few spots in the Cosmic Microwave Background," arXiv:0905.3324 [astro-ph.CO], submitted to Phys. Rev. D

[2] R. K. Sachs and A. M. Wolfe, "Perturbations of a cosmological model and angular variations of the microwave background,” Astrophys. J. 147 (1967) 73 [Gen. Rel. Grav. 39 (2007) 1929].

[3] G. Hinshaw et al. [WMAP Collaboration], "Three-year Wilkinson Microwave Anisotropy Probe (WMAP) observations: Temperature analysis,” Astrophys. J. Suppl. 170, 288 (2007) [arXiv:astro-ph/0603451].

[4] M. R. Nolta et al. [WMAP Collaboration], "Five-Year Wilkinson Microwave Anisotropy Probe (WMAP) Observations: Angular Power Spectra,” Astrophys. J. Suppl. 180, 296 (2009) [arXiv:0803.0593 [astro-ph]].

[5] A. de Oliveira-Costa, M. Tegmark, M. Zaldarriaga and A. Hamilton, "The significance of the largest scale CMB fluctuations in WMAP," Phys. Rev. D 69, 063516 (2004) [arXiv:astro-ph/0307282].

[6] K. Land and J. Magueijo, Phys. Rev. Lett. 95, 071301 (2005) [arXiv:astro-ph/0502237].

[7] C. J. Copi, D. Huterer, D. J. Schwarz and G. D. Starkman, arXiv:0808.3767 [astro-ph].

[8] P. Vielva, E. Martinez-Gonzalez, R. B. Barreiro, J. L. Sanz and L. Cayon, Astrophys. J. 609, 22 (2004) [arXiv:astro-ph/0310273].

[9] K. T. Inoue and J. Silk, Astrophys. J. 648, 23 (2006) [arXiv:astro-ph/0602478].

[10] K. T. Inoue and J. Silk, Astrophys. J. 664, 650 (2007) [arXiv:astro-ph/0612347].

[11] Z. Hou, A. J. Banday and K. M. Gorski, arXiv:0903.4446 [astro-ph.CO].

[12] D. L. Larson and B. D. Wandelt, arXiv:astro-ph/0505046.

[13] D. L. Larson and B. D. Wandelt, Astrophys. J. 613, L85 (2004) [arXiv:astro-ph/0404037].

[14] K. M. Gorski, E. Hivon, A. J. Banday, B. D. Wandelt, F. K. Hansen, M. Reinecke and M. Bartelman, Astrophys. J. 622, 759 (2005) [arXiv:astro-ph/0409513].

[15] B. Gold et al. [WMAP Collaboration], Astrophys. J. Suppl. 180 (2009) 265 [arXiv:0803.0715 [astro-ph]].

[16] A. Bernui and M. J. Reboucas, Phys. Rev. D 79 (2009) 063528 [arXiv:0806.3758 [astro-ph]].

[17] A. Hajian, arXiv:astro-ph/0702723. 